\title{
ACTIVITÉS BIOLOGIQUES DES EXTRAitS AqueuX de PERGularia tomentosa L. (ASCLEPIADACEAE)
}

R. Cherif ${ }^{1}$, A. Kemassi ${ }^{1,2}$, Z. Boual' ${ }^{2}$, N. Bouziane ${ }^{2}$, F. Benbrahim², A. Hadjseyd ${ }^{2}$, T. Gharib $^{1}$, A. Ould el Hadj-Khelil ${ }^{2}$, M.L. Sakeur ${ }^{2}$ et M.D. Ould el Hadj ${ }^{2}$

${ }^{1}$ Département de biologie, université de Ghardaïa, B.P. 455 Ghardaïa 47000, Algérie ${ }^{2}$ Laboratoire de protection des écosystèmes en zones arides et semi-arides, université Kasdi Merbah Ouargla, B.P. 511 Ouargla 30000, Algérie akemassi@yahoo.fr

(Received 14 April 2015 - Accepted 10 September 2015)

\section{RÉSUMÉ}

Cherif R., A. Kemassi, Z. Boual, N. Bouziane, F. Benbrahim, A. Hadjseyd, T. Gharib, A. Ould el Hadj-Khelil, M.L. Sakeur et M.D. Ould el Hadj. 2016. Activités biologiques des extraits aqueux de Pergularia tomentosa L. (Asclepiadaceae). Lebanese Science Journal, 17(1): 25-35.

La présente étude porte sur la recherche de l'activité allélopathique de l'extrait aqueux obtenu par reflux de Pergularia tomentosa Ait. (Asclepiadaceae) récoltée dans le Sahara septentrional Est algérien, via l'examen de ses effets sur la germination des graines d'orge Hordeum vulgare L. L'étude réalisée permet de mettre en évidence l'effet inhibiteur sur la germination des graines d'orge traitées. Les extraits aqueux purs et dilués à $50 \%$ présentent une capacité inhibitrice exceptionnelle de l'ordre de 100\%. Elle est moindre pour les concentrations à dosage assez faible. Pour des concentrations $\leq 20 \%$, l'extrait racinaire de cette plante semble plus phytotoxique que l'extrait foliaire. En outre, des anomalies de croissance sont observées au niveau des lots traités.

Mots-clés: allélopathie, extrait, Pergularia tomentosa, phytotoxicité, anomalie, Sahara

\section{ABSTRACT}

Cherif R., A. Kemassi, Z. Boual, N. Bouziane, F. Benbrahim, A. Hadjseyd, T. Gharib, A. Ould el Hadj-Khelil, M.L. Sakeur et M.D. Ould el Hadj. 2016. Biological activity of aqueous extracts of Pergularia tomentosa L. (Asclepiadaceae). Lebanese Science Journal, 17(1): 25-35.

This study focuses on the allelopathic potential of the aqueous extract, obtained under reflux conditions, of Pergularia tomentosa L. (Asclepiadaceae) harvested from the northeastern region of Algerian Sahara, through the examination of their effects on seeds germination of barley (Hordeum vulgare L.). The results obtained highlighted the inhibitory effect on seed germination of treated barley. It appeared that the pure aqueous extracts and the 50\% diluted solution have an excellent capability to inhibit the germination of barley seeds, which corresponds to a $100 \%$ inhibition rate, with reduced inhibition for lower concentrations. It is 
found that the root extract seems more phytotoxic than the foliar extract of the same plant. In addition, abnormal growth abnormalities were observed in the treated samples.

Keywords: allelopathy, extract, Pergularia tomentosa, phytotoxicity, abnormality, Sahara

\section{INTRODUCTION}

Le Sahara est caractérisé par des conditions édaphoclimatiques très contraignantes pour la survie spontanée des êtres vivants. Néanmoins, cet écosystème reste un milieu vivant caractérisé par un couvert végétal très diversifié, discontinu et très irrégulier. Les plantes utilisent surtout les emplacements où l'eau est un peu plus accessible qu'ailleurs. En particulier celle du Sahara, la végétation en zones arides et semi arides, est très clairsemée, à aspect en général nu et désolé. Les arbres sont aussi rares que dispersés et les herbes n'y apparaissent que pendant une période très brève de l'année, quand les conditions deviennent favorables (Chehma et al., 2005). La connaissance, la classification, la caractérisation et la conservation des différents taxons est une priorité scientifique mondiale pour l'évaluation et la gestion de la biodiversité (Cotterill, 1995).

La plante Pergularia tomentosa Ait. appartient à la famille des Asclepiadaceae (Maman, 2003). C'est un arbrisseau vivace, spontané, xérophyte et chamaephyte très répandue en Afrique du Nord, plus commune dans le Sahara algérien (Al-Said et al., 1989). Elle est observée en pieds isolés ou en petits groupes dans les oueds sablo-argileux et les regs, montrant une amplitude assez large pour les sols sableux, argileux graveleux ou pierreux ainsi que sur les plateaux caillouteux (Quezel, 1962 \& Santa, 1962-1963).

L'usage intensif des herbicides de synthèse, provoque des effets graves et destructeurs pour l'environnement. L'utilisation continue des herbicides pour le contrôle des adventices pose beaucoup de problèmes et constitue une préoccupation majeure pour les scientifiques, particulièrement aprés l'apparition de certaines formes de résistance chez quelques espèces adventices. Pour cela, la recherche des bio-herbicides qui peuvent constituer une solution alternative, est une nécessité certaine. Beaucoup d'auteurs ont rapporté les possibilités d'employer les extraits des plantes pour contrôler les mauvaises herbes (Siddiqui et al., 2009; Naseem et al., 2009; Hassan et al., 2012; Sahu \& Devkota, 2013).

Dans le cadre de la recherche des molécules bioactives, d'origine végétale et efficaces dans la lutte contre les mauvaises herbes, il est souhaitable d'étudier les potentialités de la flore saharienne en vue de sa valorisation. La présente recherche se propose d'examiner les activités biologiques des extraits des espèces végétales du Sahara septentrional Est algérien, sur la plante test (Hordeum vulgare $\mathrm{L}$.).

\section{MATÉRIELS ET MÉTHODES}

\section{Matériel végétal}

Le matériel végétal, constitué d'une partie aérienne et souterraine de l'espèce Pergularia tomentosa Ait. est récolté en février 2013 dans l'Oued Metlili, wilaya de Ghardaïa (Est d'Algérie). Il a été séché à l'ombre avant d'ètre broyé. 
La préparation des extraits est réalisée selon la méthode d'extraction par reflux en milieu hydroalcoolique.

\section{Plante test}

L'orge en graine était utilisé comme plante test pour vérifier le potentiel inhibiteur de la germination des extraits de Pergularia tomentosa. Les graines d'orge sont choisies en raison de leur faculté germinative élevée, pour la facilité de manipulation au laboratoire. Les graines utilisées pour l'expérimentation proviennent de la banque des semences du laboratoire de protection des écosystèmes en zones arides et semi-arides de l'université de Ouargla. Il s'agit des semences certifiées.

\section{Méthode d'extraction}

Les parties récoltées ont été séchées à l'air libre à température ambiante pendant trois à quatre jours avant d'être réduites en poudre à l'aide d'un broyeur à lame. $50 \mathrm{~g}$ de poudre végétale ont été introduits dans un erlenmeyer, contenant $1 / 3$ d'eau distillé et $2 / 3$ de méthanol. L'ensemble a été porté à ébullition pendant six heures selon le protocole d'extraction par reflux. La suspension a été ensuite filtrée à chaud successivement sur papier filtre dans un flacon en verre. Le filtrat recueilli est évaporé sous pression réduite afin d'éliminer le méthanol. Le résidu récupéré est utilisé pour les tests biologiques.

\section{Constitution des lots expérimentaux}

L'extrait de la plante espèce (Pergularia tomentosa Ait.) a été utilisé soit à l'état pur, ou à des concentrations décroissantes $(100 \%, 50 \%, 25 \%, 20 \%, 15 \%, 10 \%, 05 \%, 2,5 \%$ et $01 \%$ ), soit au total, 9 concentrations différentes. Les graines de la plante test (orge), au nombre de dix, sont déposées dans une boîte de Pétri, Elles sont irriguées le premier jour par $3 \mathrm{ml}$ de l'extrait végétal ou témoin, et, par la suite, quotidiennement par $1 \mathrm{ml}$ d'eau distillée.

L'expérimentation est suivie durant 10 jours, tout en respectant le protocole expérimental et en notant quotidiennement le nombre de graines germées, qui servent par la suite aux analyses de la cinétique de la germination observée au niveau des différents lots constitués.

Afin d'accomplir ce travail, différents paramètres sont examines.

\section{Paramètres étudiés}

\section{Taux d'inhibition}

La capacité d'une substance ou préparation à inhiber la germination des graines est exprimée par la relation suivante (Ben Khettou, 2010):

$\mathrm{N}_{\mathrm{s}}$ : Nombre de graines semées.

$$
T_{i}=\frac{\left(\mathrm{N}_{\mathrm{s}}-\mathrm{N}_{\mathrm{g}}\right)}{\mathrm{N}_{\mathrm{s}}} .100
$$

$\mathrm{N}_{\mathrm{g}}$ : Nombre de graines germées. 


\section{Taux de germination}

Selon Côme (1970), le taux de germination correspond au pourcentage des graines germées par rapport au total des graines semées, il est estimé par la formule suivante:

$\mathrm{N}_{\mathrm{g}}$ : Nombre de graines germées.

$$
T_{g}=\frac{\mathrm{N}_{\mathrm{g}}}{\mathrm{N}_{\mathrm{s}}} \cdot 100
$$

$\mathrm{N}_{\mathrm{s}}$ : Nombre de graines semées.

\section{Cinétique de germination}

La cinétique de germination correspond aux variations dans le temps du taux de germination des graines de la plante test. Elle représente graphiquement le pourcentage de germination en fonction du temps. Elle donne une vision précise sur l'évolution de germination d'un lot de semences placé dans des conditions bien déterminées (Côme, 1970).

\section{Vitesse de germination}

D'après Côme (1970), la vitesse de germination peut être exprimée de plusieurs façons :

-Pourcentage de semences germées ou taux de germination au bout d'un certain temps après l'ensemencement;

-Le temps moyen nécessaire à la germination représente l'inverse du «Coefficient de vélocité» (Kotowisk, 1926; Ben Khattou, 2010).

$$
C_{v}=\frac{N_{1}+N_{2}+\ldots \ldots+N_{n}}{\left(N_{1} T_{1}+N_{2} T_{2}+\ldots \ldots+N_{n} T_{n}\right)} \cdot 100
$$

$\mathrm{N}_{1}$ : nombre de graines germées au temps $\mathrm{T}_{1}$

$\mathrm{N}_{2}$ : nombre de graines germées au temps $\mathrm{T}_{2}$.

$\mathrm{N}_{\mathrm{n}}$ : nombre de graines germées au temps $\mathrm{T}_{\mathrm{n}}$.

\section{Concentration d'efficacité}

C'est la quantité d'une matière administrée en une seule fois, causant la mort de $50 \%$ (la moitié) d'un groupe traité ou bien donnant $50 \%$ de succès. La CE50\% est une façon de mesurer le potentiel toxique à court terme (toxicité aiguë) d'une matière. C.E. (exp. C.E.50\%); concentration efficace qui inhibe un pourcentage donné d'une réponse biologique, de type binaire (exp. germination ou absence de germination) (Côme, 1970).

\section{Index de germination}

D'après Throneberry et Smith (1955), l'index de la germination est une expression quantitative de la germination qui concerne le taux de germination quotidien à la valeur maximale de la germination notée. Il est donné par la relation suivante:

$\mathrm{N}_{\mathrm{n}}$ : est le pourcentage de germination obtenu au nième jour.

$$
I_{g}=N_{1}+\frac{\left(N_{2}-N_{1}\right)}{2}+\frac{\left(N_{3}-N_{2}\right)}{3}+\cdots+\frac{\left(N_{n}-N_{n-1}\right)}{n}
$$




\section{RÉSULTATS ET DISCUSSION}

\section{Taux d'inhibition}

Au vu des résultats obtenus et résumés (Figure 1), on peut noter que l'extrait végétal foliaire ou racinaire pur ou dilué à $50 \%$ présente une capacité exceptionnelle à inhiber la germination des graines de la plante test. Par contre, chez l'extrait foliaire dilué à $25 \%$, le taux d'inhibition de la germination est de 30\%. Pour les concentrations inférieures (20\%-15\%), le taux d'inhibition maximal enregistré oscille entre $16,67 \%$ et $3,33 \%$. Comparativement à l'extrait racinaire, le taux d'inhibition maximum est de 23,33\%. Pour les dilutions allant de 20 à $15 \%$, le taux d'inhibition maximum enregistré varie de $13,33 \%$ à 3,33\%. Généralement, on note que l'extrait racinaire possède une capacité d'inhibition de la germination plus efficace que celle de l'extrait foliaire. Cette action est probablement liée à la concentration des extraits en molécules actives capables d'empêcher la germination des graines. Il est admis que dans les conditions naturelles, la germination des graines est un processus biochimique et physiologique où dès le premier contact de la graine avec le stimulus exogène (eau), une enzyme amylase est synthétisée et secrétée afin de dégrader l'amidon (albumine) en vue de fournir à l'embryon l'énergie nécessaire à la germination (Regnault-Roger et al., 2008). Une fois secrétée, la croissance embryonnaire s'amorce et intervient par la suite par un autre processus physiologique où les acteurs sont les hormones de croissance végétale dont l'auxine, par exemple (Lesuffleur, 2007).

\section{Taux maximum de germination}

D'après les résultats de la Figure 2, on a constaté que l'extrait aqueux foliaire ou racinaire pur ou dilué à $50 \%$ engendre une inhibition totale de la germination des graines d'orge après 10 jours de suivi quotidien. Selon les résultats obtenus, le taux maximum de germination est de $70 \%$ au niveau du lot traité par l'extrait foliaire à $25 \%$. Alors que pour les autres concentrations, les taux de germination signalés varient entre 83,33\% à 93,33\%. Par contre, pour l'extrait racinaire, un pourcentage de germination de $76,67 \%$ est noté pour l'extrait dilué à $25 \%$. Ce pourcentage varie entre $86,67 \%$ à $93,67 \%$ pour les autres concentrations. De ce fait, la capacité à inhiber la germination des graines est un processus complexe, où plusieurs hypothèses peuvent être établies dont la capacité de certaines molécules qui se trouvent dans les extraits à inhiber l'action de l'enzyme amylase ou bien d'occuper leurs sites membranaires, ou bien à l'action mimétique ou antagoniste de ces molécules vis-à-vis des hormones de croissance, ou à l'inhibition de leurs actions tissulaires (Feeny, 1976).

\section{Cinétique de la germination}

Selon les deux Figures 3 et 4, nous avons remarqué une variation dans le taux de germination journalier observé au niveau du lot témoin par rapport aux lots traités. Au niveau du lot témoin, aucune germination $\mathrm{n}$ a' été observée dans le premier jour de l'expérimentation. Après 48 heures, un taux de germination de $66,67 \%$ et $73,33 \%$ est enregistré chez les graines

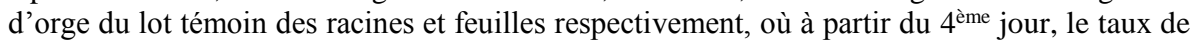
germination est atteint. Alors que pour les espèces adventices tests, la germination a commencé dès le $2^{\text {ème }}$ jour pour les graines d'orge traitées par l'extrait foliaire et racinaire dilué à $10 \%, 5 \%, 2,5 \%$ et $1 \%$, et dès le $3^{\text {ème }}$ jour pour les lots traités par les extraits dilués à $25 \%$ et $20 \%$. Pour les autres traitements dont les extraits à $100 \%$ et $50 \%$, aucun cas de germination 
n'est observé. D'après Evenari (1957), la germination est considérée comme étant le passage d'une semence inerte (vie ralentie) à une jeune plantule autotrophe.

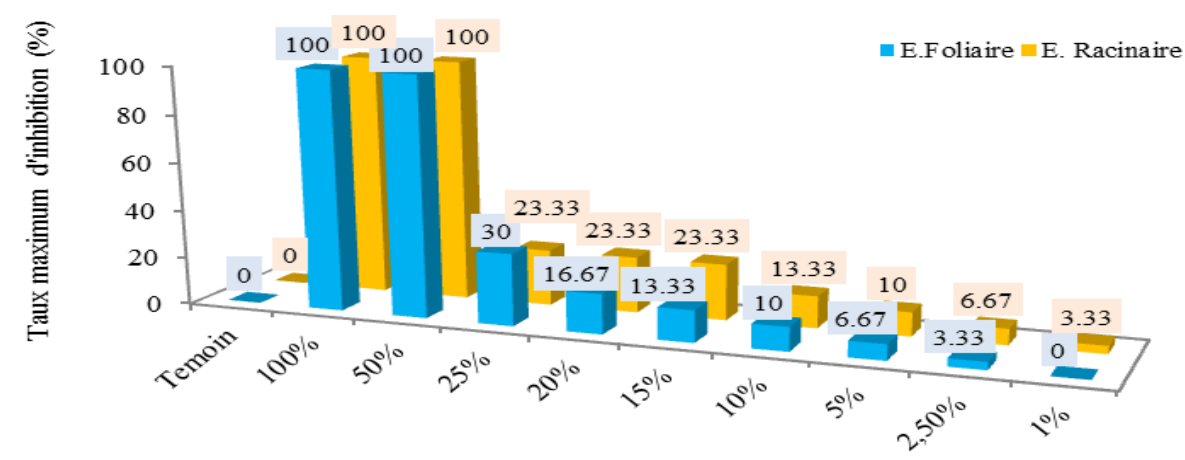

Lots expérimentaux

Figure 1. Taux d'inhibition maximum de germination enregistré au niveau des différents lots témoins et traités par l'extrait foliaire et racinaire aqueux de Pergularia tomentosa Ait.

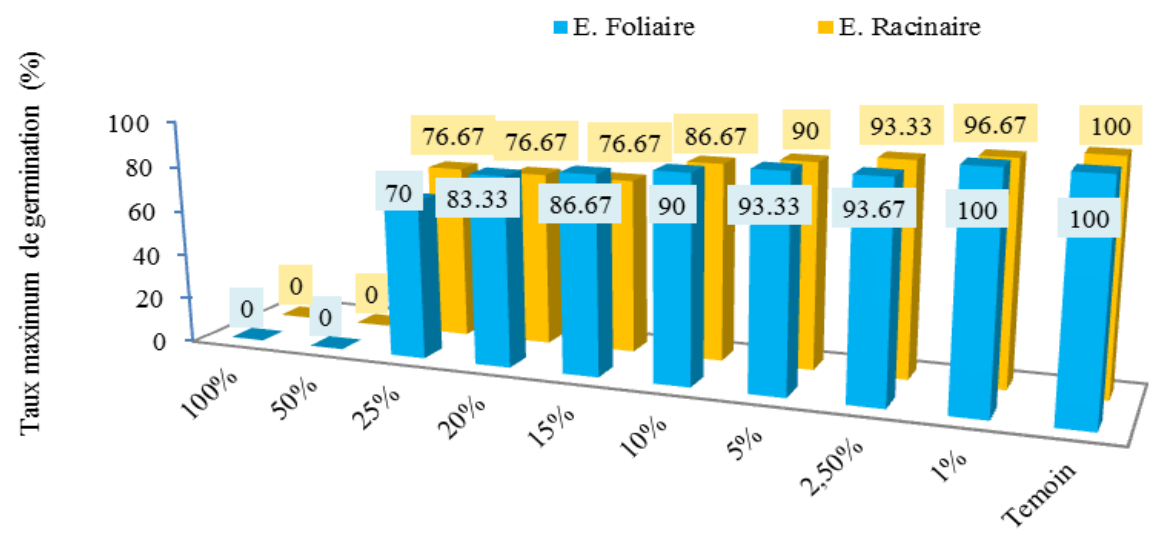

Lots expérimentaux

Figure 2. Taux maximum de germination enregistré au niveau des différents lots témoins et traités par l'extrait foliaire et racinaire aqueux de Pergularia tomentosa Ait. 


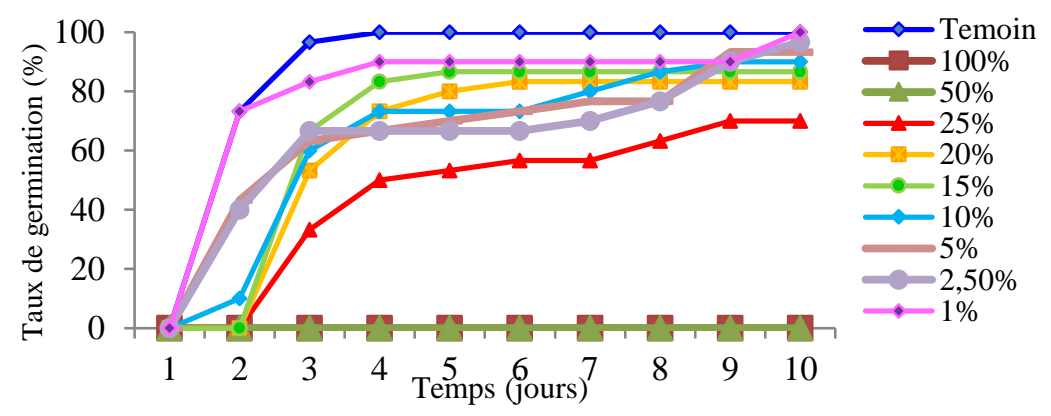

Figure 3. Cinétique de la germination observée au niveau des différents lots témoins et traités par l'extrait racinaire aqueux de Pergularia tomentosa Ait.

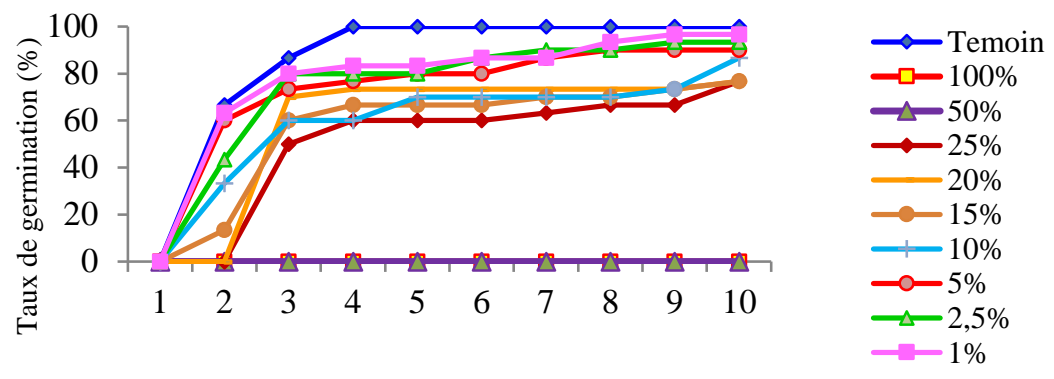

Temps (jours)

Figure 4. Cinétique de la germination observée au niveau des différents lots témoins et traités par l'extrait foliaire aqueux de Pergularia tomentosa Ait..

Les processus physiologiques qui se déroulent pendant cette phase sont très complexes. Cependant, l'activité peut se mesurer par le biais de plusieurs facteurs, principalement: imbibition et respiration.

En effet, une inhibition de la synthèse d'ADN dans les noyaux du méristème apical et des racines est soupçonnée (Koitababashi et al., 1997). Cette inhibition a été citée également par (Regnault-Roger et al., 2008). La synthèse des protéines et des acides nucléiques peut aussi être effectuée par plusieurs composés phénoliques qui ralentissent l'incorporation des acides aminés (Cameron \& Julian, 1980). Des composés phénoliques peuvent être impliqués dans le contrôle de l'activité des hormones végétales. La suppression de la dégradation de l'acide indole acétique (AIA) par différents phénols a ainsi été rapportée par Lee et al. (1982). La synthèse ou le fonctionnement de plusieurs enzymes liées à la croissance sont aussi parfois perturbés (Sato et al., 1982). En outre, les alcaloïdes, flavonoïdes ont la capacité d'inhiber l'action de certaines enzymes végétales, telles que ATPase, ou bloquer le déroulement de certains phénomènes, tels que la phosphorylation, le métabolisme oxydatif, le transport membranaire, la réduction de la synthèse de certaines protéines et lipides. D'autres travaux ont montré l'action de quelques métabolites secondaires, comme, les benzoxazolinones, substances inhibitrices de l'auxine du coléoptile de l'avoine (Bais et al., 2004). 


\section{Vitesse de germination}

Au vu des résultats de la Figure 5, il ressort que pour les deux traitements (extrait pur et dilué à 50\%), aucune germination n'à été rapportée. La vitesse de germination varie entre 14,44 et 16,28 graines/jour pour les autres concentrations. Donc, quelle que ce soit la concentration en extrait foliaire ou racinaire de Pergularia tomentosa Ait. appliquée, la vitesse de germination des graines d'Hordum vulgare L. est plus faible que celle rapportée pour le lot témoin.

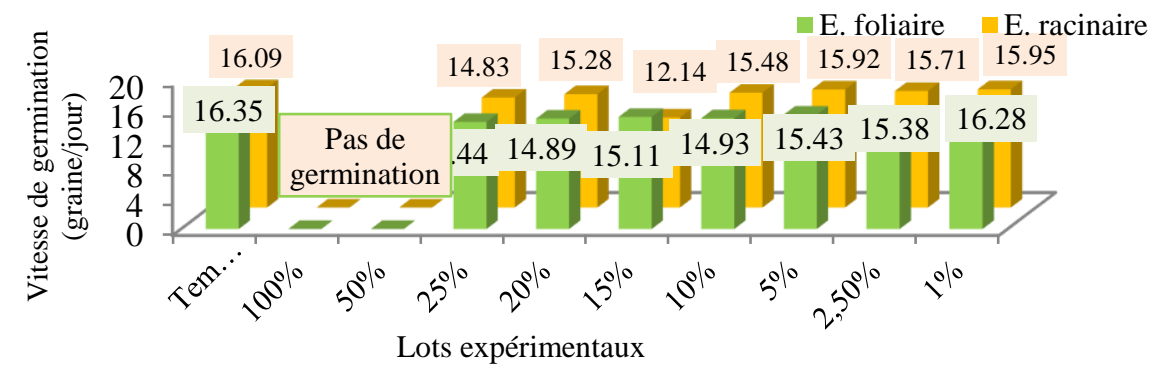

Figure 5. Vitesse de germination des graines d'orge au niveau des différents lots témoins et traits.

$\mathrm{Du}$ point de vue physiologique, la germination comme est un processus dont les limites sont le début de l'hydratation de la semence et le tout début de la croissance de la radicule. D'autre part, Côme (1970) note qu'une semence est considérée comme germée, lorsque la radicule a percé les enveloppes, ou s'il s'agit d'un embryon nu, lorsque la radicule est visiblement allongée.

\section{Concentration d'efficacité}

Le Tableau 1 regroupe les valeurs de la $\mathrm{CE}_{50 \%}$ et $\mathrm{CE}_{90 \%}$ calculées pour les deux extraits (foliaire et racinaire). Il est constaté que l'extrait obtenu à partir des racines de $P$. tomentosa est plus efficace sur la germination des graines d'orge comparativement à l'extrait foliaire de cette même plante. La $\mathrm{CE}_{50 \%}$ notée pour l'extrait racinaire étant plus faible, elle est de l'ordre de $0,0081 \mathrm{mg} / \mathrm{ml}$ et de $0,012 \mathrm{mg} / \mathrm{ml}$ pour l'extrait aqueux foliaire de $P$. tomentosa vis-àvis des graines d'orge.

\section{TABLEAU 1}

Concentrations d'Efficacités (CE50\%, CE90\%) des Extraits Végétaux de Pergularia tomentosa Ait.

\begin{tabular}{|c|c|c|c|}
\hline \multirow{2}{*}{$\begin{array}{l}\text { Extrait de } P \text {. tomento- } \\
\qquad s a\end{array}$} & \multirow{2}{*}{ Espèce test } & \multicolumn{2}{|c|}{ Concentration d'efficacité $[\mathrm{mg} / \mathrm{ml}]$} \\
\hline & & $\mathrm{CE}_{50 \%}$ & $\mathrm{CE}_{90 \%}$ \\
\hline Foliaire & $H=H_{0}$ & 0,012 & 0,029 \\
\hline Racinaire & & 0,0081 & 0,028 \\
\hline
\end{tabular}


La variation dans les valeurs des concentrations d'efficacité (CE50\%) rapportée entre les deux extraits vis-à-vis des graines d'orge émane de la variabilité dans la composition chimique entre les deux extraits, alors que celle observée pour le même extrait vis-à-vis de la plante test est probablement due à la différence dans la structure, la biologie et la taille des graines de la plante test. Chez les végétaux, la croissance est un phénomène fortement influencé par les conditions exogènes (biotiques et abiotiques).

\section{Index de germination}

D'après les résultats obtenus dans le Tableau 2, il est observé qu'au niveau des lots purs et dilués à $50 \%$, aucun cas de germination n'a été enregistré. Dans les cas où la concentration est faible (de $25 \%$ à $1 \%$ ), l'index de la germination se trouve en augmentation, et ce, en fonction de la diminution de la concentration. Pour cela, il est à déduire quelle que soit la concentration en extrait foliaire ou racinaire (Pergularia tomentosa Ait.), l'index de la germination est plus faible que celui du lot témoin.

Certains métabolites secondaires végétaux influent sur la germination ou la croissance des plantes par des mécanismes multiples (Einhellig, 1985). La division et l'élongation cellulaire, phases essentielles pour le développement, sont sensibles à la présence des composés allélopathiques (Muller, 1965).

TABLEAU 2

Index de Germination des Plantules d'Orge Témoins et Traités

Lots expérimentaux

\begin{tabular}{|c|c|c|c|c|c|c|c|c|c|c|}
\hline & \multicolumn{10}{|c|}{ Lots experimentaux } \\
\hline & Témoin & $100 \%$ & $\mathbf{5 0 \%}$ & $25 \%$ & $20 \%$ & $15 \%$ & $10 \%$ & $5 \%$ & $2,5 \%$ & $1 \%$ \\
\hline E. foliaire & 46,94 & I & I & 18,10 & 24,10 & 27,04 & 27,14 & 32,91 & 32,67 & 42,65 \\
\hline E. racinaire & 43,63 & / & I & 21,04 & 24,96 & 25,14 & 29,25 & 37,29 & 35,83 & 39,79 \\
\hline
\end{tabular}

Il est admis que les substances de croissance végétales dont les auxines sont synthétisées dans les apex caulinaires et racinaires et transportées dans l'axe de la plante. L'allongement des racines est particulièrement sensible à l'auxine (AIA) qui, à des très faibles concentrations, provoque la croissance des racines excisées ou intactes, et à des concentrations plus élevées, elles stimulent l'allongement des tiges en inhibant fortement la croissance des racines (Hopkins, 2003).

\section{CONCLUSION}

Le présent travail de recherche a pour objectif de mettre en évidence l'action des extraits aqueux de Pergularia tomentosa Ait. sur la germination des graines d'orge (Hordeum vulgare $\mathrm{L}$.). 
Les extraits utilisés sont d'origine foliaire et racinaire, récoltés dans le Sahara septentrional à Oued Metlili (Est Algérien). Plusieurs tests ont été effectués, à différentes concentrations, soit à $100 \%, 50 \%, 25 \%, 20 \%, 15 \%, 10 \%, 5 \%, 2,5 \%$ et $1 \%$. D'après les résultats des analyses, il a été constaté en premier lieu que les extraits aqueux purs et dilués à 50\% présentent un effet inhibiteur significatif sur la germination des graines d'orge. Aucun signe de germination, en effet, n'a été constaté. Il s'agit d'une inhibition totale (100\%). Dans le cas d'une dilution (faible dose), l'effet inhibiteur est quasi-partiel. Donc, l'extrait aqueux empêche la germination des graines comparativement aux graines du lot témoin. Aussi, ces résultats mettent en évidence l'effet allélopathique ou phytotoxique des extraits aqueux de cette plante vis-à-vis des graines d'orge. En plus, il est remarqué que tous les extraits appliqués à l'état pur, présentent un effet inhibiteur significatif de la germination. Lorsqu'ils sont appliqués à des concentrations relativement faibles l'effet inhibiteur est fortement affecté. Certains signes de germination ont été relevés. Ces observations ont été prouvées par les résultats de l'étude, portant sur l'effet inhibiteur de la germination par effet choc, où les extraits sont appliqués une fois sans répétition et le taux d'inhibition est de $100 \%$.

À la lumière de ces résultats, il est intéressant de conclure que les extraits aqueux de Pergularia tomentosa Ait. possèdent un effet d'inhibiteur agissant même à faibles concentrations et empêchent ainsi la germination des graines d'orge. De ce fait, une meilleure connaissance de ce phénomène pourrait offrir des perspectives intéressantes pour la gestion de la flore spontanée des parcelles cultivées, et ainsi contribuer à diminuer l'utilisation d'herbicides de synthèse.

\section{REFERENCES}

Al-Said, M.S., Abu-Jayyab, A. et Hifnawy, M.S. 1989. Biochemical studies on ghalakinoside, a possible antitumor agent from Pergularia tomentosa Ait. Journal of Ethnopharmacology, 27(1/2): 235-240.

Bais, H.P., Vepachedu, S., Gilroy R.M., Callaway, R.M. et Vivanco, J.M. 2003. Allelopathy and exotic plant invasion: form molecules and genes to species interactions. Science, 301: 1377-1380.

Bais, H.P., Park, S.W., Weir, T.L., Callaway, R.M., Vivanco, J.M. 2004. How plants communicate using the underground information superhighway. Trends Plant Sci., 9: 26-32. doi: 10. 1016/ j. tplants.2003.11.008.

Baziramakenga, R., Leroux, G.D., Simard, R.R. 1994. Effects of benzoic and cinnamic acids on growth, mineral composition and chlorophyll content of soybean. Journal of Chemical Ecology, 20(11): 2821-2833.

Ben Khettou, H. 2010. Contribution à l'étude de l'aptitude à la germination des graines d'Agrania spinosa L. (SAPOTACEAE) dans la région d'Ouargla. Mémoire ing. éco., univ. Ouargla.

Cameron, H.J. and Julian, G.R. 1980. Inhibition of protein synthesis in lettuce (Latuca sativa L.) by allelopathic compounds. Journal of Chemical Ecology, 6(6): 989-995.

Chehma, A., Djebar, M.R., Hadjaiji, F., Rouabeh, L. 2005. Étude floristique spatio-temporelle des parcours sahariens du Sud- Est algérien. Sécheresse, 16(4): 275-285.

Come, D. 1970. Les obstacles à la germination (Monographie et physiologie végétale $\left.n^{\circ} 6\right)$. Éd. Masson et Cie (Paris), pages 14, 24 et 27.

Cotterill, F.P.D. 1995. Systematic biological knowledge and environmental conservation. Biodiversity and Conservation, (4): 183-205. 
Einhellig, F.A. 1985. Effects of allelopathic chemicals on crop productivity. In: Bioregulators for pest control. Ed. P.A. Heldin, ACS Symp. Ser. 276, Amer. Chem. Soc., Washington, DC, pp. 109-130.

Évenari, M. 1957. Les problèmes physiologiques de la germination. Bulletin société française physiologie végétale, 3(4): 105-124.

Feeny, P. 1976. Plant appetency and chemical defense. Ed. Plenum Press, New York.

Hopkins, W.G. 2003. Physiologie végétale. Boeck et Larcier, Bruxelles, 267-283p.

Koitababashi, R., Suzuki, T., Kawazu, T., Sakai, A., Kuroiwa, H. et Kuroiwa, T. 1997. 1,8cineole inhibits root growth and DNA synthesis in the root apical meristem of Brassica campestris L. J. Plant Res., 110: 1-6.

Kotowski, F. 1926. Temperatures relations to germination of vegetable seed. Proc. Amer. Soc. Horticult. Sei., 23: 176-184 pages.

Lee, T.T., Staratt, A.N., Jevnikar, J.J. 1982. Regulation of enzymic oxidation of indole-3acetic acid by phenols; structure activity relationship. Phytochemistry, 21: 517-523.

Lesuffleur, F. 2007. Rhizdéposition à court terme de l'azote et exsudation racinaire des acides aminés par le tréfle blanc (Trifoluim repense L.). 17-37p.

Hassan, M.M., Daffalla, M.H., Yagoub, O.S., Osman, M.G., Abd el Ghani, M.G. and Babiker, E.A. 2012. Allelopathic effects of some botanical extracts on germination and seedling growth of Sorghum bicolor L. Journal of Agricultural Technology, 8(4): 1423-1469.

Muller, C.H. 1965. Inhibitory terpenes volatilized from Salvia shrubs. Bulletin of the Torrey Botanical Club, 92: 38-45.

Naseem, M., Aslam, M., Ansar, M. and Azhar, M. 2009. Allelopathic effects of sunflower water extract on weed control and wheat productivity. Pakistan Journal of Weed Science Research, 15(1): 107-116.

Quezel, P. et Santa, S. 1962-1963. Nouvelle Flore de l'Algérie et des régions désertiques méridionales. CNRS, Paris, 2 vols., 1170 pages.

Regnault-Roger, C., Philogene, B.Jr. et Vincent, Ch. 2008. Bio-pesticides d'origine végétale. Ed. TEC \& DOC, Paris, p. 51-60.

Sahu, A. and Devkota, A. 2013. Allelopathic effects of aqueous extract of leaves of Mikania micrantha H.B.K. on seed germination and seedling growth of Oryza sativa L. and Raphanus sativus L. Scientic World, 11(11): 90-93.

Sato, T., Kiuchi, F., Sankaxa, U. 1982. Inhibition of phenyl-alanine-ammonia-lyase by cinnamic derivatives and related compounds. Phytochemistry, 21: 845-850.

Siddiqui, S., Bhardwaj, S., Saeed Khan, S. and Kumar Meghvanshi, M. 2009. Allelopathic effect of different concentration of water extract of Prosopsis juliflora leaf on seed germination and radicle length of wheat (Triticum aestivum Var-Lok-1). AmericanEurasian Journal of Scientific Research, 4(2): 81-84.

Throneberry, G.O. et Smith, F.G. 1955. Relation of respiratory and enzymatic activity to corn seed viability. Plant Physiology, 30: 337-343. 